\title{
The analysis of the prosperity indexes for Guangxi's electricity market ${ }^{*}$
}

\author{
Li-Juan Qin, Xu Yan, Shuai Han, Xi-Qiao Lin, \\ Bo Zeng and Li-Ke Gao \\ Electric Power Research Institute of Guangxi Power Grid Co., Ltd. \\ Nanning, Guangxi, China \\ ${ }^{\dagger}$ E-mail:qin_lj.sy@gx.csg.cn,594842366@qq.com
}

\begin{abstract}
The research object of electricity marketing is electricity consumption market. It is of great significance for judging the trend of its development and guiding the decision maker to analyze the prosperity of electricity consumption market. This paper put forward an entire system from disposal to filter the indexes of power-supply market, which formed the standardized analyzing process. It used the method of X-12-ARIMA in the seasonal decomposing and cross-correlation in the SPSS program to calculate leading-indexes and lag-indexes, forming the leading indicators, coincidence indicators, and lagging indicators. After that, the prosperity index of electricity consumption market can be made through the calculation of diffusion index and composite index. Through some examples in Guangxi, it can reflect the long-term prosperity situation and the trend of the electricity consumption market through the fluctuation of the calculated indicators to some extents
\end{abstract}

Keywords: Electricity Market; Prosperity Analysis; Diffusion Index; Composite Index.

\section{Introduction}

"Boom", which is the abbreviation of the economic boom, is a concept to describe the economy is in a high active stage in western developed countries. The economic analysis and research originated from the economic field, and its application is expanding to other areas. According to the characteristics of electricity demand, based on the diffusion index method and the composite index method, the economic analysis method calculates the diffusion index and composite index of leading indicators, consistent indicators and lagging indicators to prepare the sentiment index of target market.

Literature [1]-[5] introduced the economic analysis in the field of research and application of the situation respectively from the financial, industrial,

\footnotetext{
* This work is supported by science and technology project of Guangxi Power Grid Co., Ltd (GX2014-2-0019)
} 
petrochemical and other industries. The diffusion index and composite index are the foundation and core of economic analysis, which provide strong support for the fluctuation range and the change trend of quantitative indicators[1,3,5]. Tie Mei Gao and Wen Fang Liang apply the boom analysis of synthetic index method to above scale industrial area, build the principal component analysis model, establish the booming index, simplify the procedures for calculating the synthetic index, accurate calculation results, which greatly improve the economic analysis of the application of the scope and accuracy[3]. Literature $[7,8]$ described the sufficiency and necessity of the analysis of the prosperity of the economy in power utilization market, which is an important part of electricity market. In other words, economic analysis is not only able to quantify the power consumption of the electricity industry fluctuations and long-term trends, but also to a certain extent reflect the industry in the next period of time the power demand. The study of foreign economic analysis mainly focuses on market risk, finance, power grid stability boundary, supply chain management, decision support, project management, reliability and environment, etc.

In this paper, the effects of extreme data, Spring Festival effect and holiday effect are eliminated by seasonal adjustment. The lead time and the lag period of each index are calculated, and the first index group, the consistent index group and the lagging index group are constructed respectively. Then, calculate the index group which has already been established by the method of diffusion index and principal component analysis, making use of the electricity market prosperity index to realize the advance judgment of the long-term change trend of regional electricity market.

\section{The Processing of Sentiment Analysis Indicators}

\subsection{The principle and method of X-12-ARIMA seasonal adjustment}

In this paper, the original data is processed by the X-12-ARIMA seasonal adjustment method, and the effect of the seasonal factor and the calendar effect is eliminated. X-12-ARIMA seasonal adjustment software implementation of the seasonal adjustment process, can be divided into regARIMA and enhanced version of the $\mathrm{X}-11$ two modules. Figure 1-1 is the basic process of the program, through the process of "adjustment - diagnosis - re adjustment", it will get the best season of seasonal adjustment.

\subsubsection{The principle and method of regARIMA modeling}

Pre adjustment module regARIMA is mainly used for data preprocessing. For a given sequence ${ }^{\gamma_{t}}$, before establishing the time series model, it is necessary to carry on the nonlinear transformation to obtain a sequence which can be fully 
fitted by regARIMA. The transformation of the program is a family of single parameter transformation.

$$
y \underset{t}{(\lambda)}= \begin{cases}Y_{t} / d_{t} & \lambda=1 \\ \lambda^{2}+\left[\left(Y_{t} / d_{t}\right)^{\lambda}-1\right] / \lambda & \lambda \neq 0,1 \\ \log \left(Y_{t} / d_{t}\right) & \lambda=0\end{cases}
$$

$\lambda=1$ corresponds to linear transformation, $\lambda=0$ corresponds to the logarithmic transformation, the remaining transformations change smoothly with A. When $\lambda$ changes smoothly, such as the general economic indicators of the sequence, it usually needs to do the logarithmic transformation. $d_{t}$ is a divisor sequence.

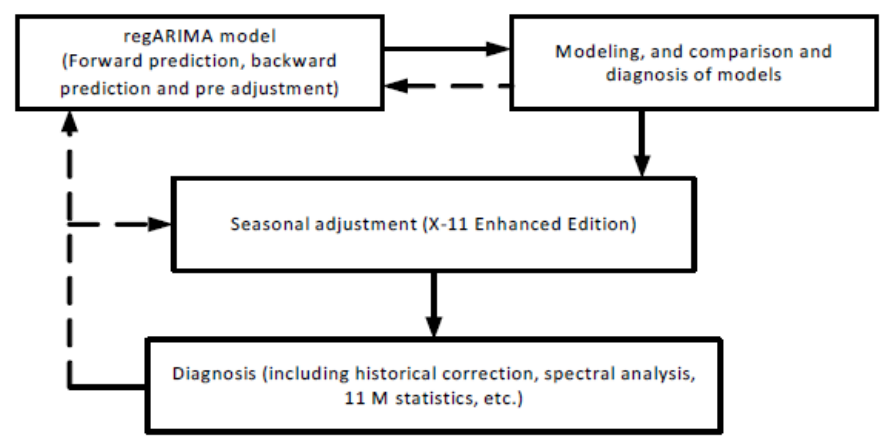

Fig. 1 Basic process of X-12-ARIMA seasonal adjustment program

ARIMA module in the regARIMA model of the general product season ARIMA model, that is, the target time series can be recorded as follows:

$$
\phi_{p}(B) \phi_{p}\left(B^{s}\right)(1-B)^{d}\left(1-B^{s}\right)^{D} z_{t}=\theta_{q}(B) \Theta_{Q}\left(B^{s}\right) a_{t}
$$

The variables in the formula are interpreted as follows:

B is hysteresis operator $\left(\boldsymbol{B} z_{t}=z_{t-1}\right) ; \boldsymbol{S}$ is seasonal period(monthly data $s=12 \quad$ quarterly data $s=4 \quad$ ); $\boldsymbol{\varphi}_{P}(\boldsymbol{B})=\left(\boldsymbol{1}-\boldsymbol{\varphi}_{\mathbf{1}} \boldsymbol{B}-\cdots-\boldsymbol{\Phi}_{P} \boldsymbol{B}^{P}\right)$ is non seasonal autoregressive operator; $\Phi_{P}(\boldsymbol{B})=\left(\mathbf{1}-\Phi_{1} \boldsymbol{B}-\cdots-\Phi_{P} \boldsymbol{B}^{P}\right)$ is seasonal autoregressive operator; $\boldsymbol{O}_{\boldsymbol{Q}}(\boldsymbol{B})=\left(1-\boldsymbol{O}_{\mathbf{1}} \boldsymbol{B}-\cdots-\boldsymbol{O}_{\boldsymbol{a}} \boldsymbol{B}^{\boldsymbol{C}}\right)$ is non seasonal moving average operator; $\Theta_{e}(\boldsymbol{B})=\left(\mathbf{1}-\Theta_{1} \boldsymbol{B}-\cdots-\Theta_{e} \boldsymbol{B}^{\mathrm{C}}\right)$ is seasonal moving average operator; $\boldsymbol{a}_{\boldsymbol{t}}$ is the white noise which is independent and identically distributed, with a mean value of 0 and a variance of $\sigma^{2}$; 
$(\mathbf{1}-\boldsymbol{B})^{d}\left(\mathbf{1}-\boldsymbol{B}^{s}\right)^{D}$ express the number of no seasonal difference is $d$, seasonal difference times are $\boldsymbol{D}$.

The sequence ${ }^{y_{t}}$, after transformation and pre adjustment, it is also required to remove outliers and calendar effects by linear regression, that is,

$$
y_{t}=\sum_{i=1}^{r} \beta_{i} x_{t}+z_{t}
$$

For $y_{t}$, the error is a sequence with ARIMA structure.

$\sum_{i=1}^{t} \beta_{i} x_{i}$ includes all exceptions and calendar effects.

\subsubsection{Enhanced version of the $X-11$ seasonal adjustment principle}

The enhanced version of the X-11 module contains three main phases, after repeated "filtration" of seasonal factors and trend factors, the final estimate of several factors is obtained. Symmetric seasonal filters and symmetric trend filters are used.

The symmetric seasonal filter expression is as follow:

$$
S_{t}^{3 \times(2 n+1)}=\frac{1}{3}\left(S_{t-12}^{2 n+1}+S_{t}^{2 n+1}+S_{t+12}^{2 n+1}\right)
$$

Among it:

$$
S_{t}^{2 n+1}=\frac{1}{2 n+1} \sum_{j=-n}^{n} S I_{t+12 j}
$$

$S_{t}^{3 \times(2 n+1)}$ is a moving average of 3 items from the SI ratio of $t$ from the same calendar month, $A$ is used to estimate seasonal factors (SI ratio) in the season.

The symmetric trend filter is used to estimate the trend of a cyclic component(TC),is a special weighted moving average:

$$
M A_{t}=\sum_{i=-H}^{H} h_{i}^{(2 H+1)} y_{t+i}
$$

According to the length of the actual sequence and the size of the irregular factors, the moving average coefficient $h_{i}^{(2 H+1)}$ is automatically selected. 


\subsection{Seasonal adjustment of the Spring Festival factors}

X-12-ARIMA needs to carry out the Spring Festival factor regression calculation. According to the date of the annual Spring Festival to determine the impact day distribution of the number of days in each month, and then determine the weight of each month, there is no impact on the date of the month weight is 0 , the weight of each year and 1 .

In the seasonal adjustment of the index, the index of assumption of Spring Festival in the Spring Festival 15 days before the 5 days after the spring festival. The weight sequence is modeled as a user self-defined regARIMA model, and the program automatically generates the regression coefficients and their $\mathrm{T}$ statistics.

\subsection{Using X-12-ARIMA seasonal adjustment method to deal with the economic analysis index}

The seasonal adjustment of the power grid electricity consumption is as follows: multiplicative model; corresponding multiplicative model, in the regARIMA and adjustment of the sequence of the logarithmic transformation of the sequence; eliminate the influence factors through the pre adjustment of leap year; automatic detection of outliers in the regARIMA link; in the regARIMA link to join the user defined regression variable estimated Spring Festival effect; ARIMA model in the automatic selection of ARIMA model, if a number of models are selected, then select the best model to predict the expansion effect; Automatic selection of season and trend filter in X-11 link.

\section{Screening of Electricity Market Sentiment Index}

\subsection{General classification method of sentiment index}

According to the time difference between the indicators, the economic indicators are divided into leading indicators, consistent indicators and lagging indicators.

-Leading indicators selection criteria are as follows:

(1)From the economic sense, the analysis of the first indicators have a clear, positive relationship.

(2)Compared with the reference cycle peak, the peak value is at least 3 months or more, with a minimum of 3 consecutive periodic fluctuations, at least two times to maintain the first, leading more than 3 months.

-Consistent index selection criteria are as follows:

(1)From the point of economic analysis, the synchronization index should have obvious characteristics of synchronization with the reference cycle. 
(2)The peak value is close to the base cycle, and the difference is within 2 months.

-Lagging indicators also have their own selection criteria:

(1)From the economic analysis, lagging indicators have a certain lag relationship.

(2)Compared with the reference cycle, the peak value should be more than 3 months.

\subsection{Time difference correlation analysis}

Delay time correlation coefficient of each indicator is calculated by the time correlation analysis method, negative numbers mean ahead, 0 express agreement, positive numbers means lag.

Set $y=\left[\mathrm{y}_{1}, \mathrm{y}_{2}, \cdots, \mathrm{y}_{\mathrm{n}}\right]$ as the reference cycle, $x=\left[\mathrm{x}_{1}, \mathrm{x}_{2}, \cdots, \mathrm{x}_{\mathrm{n}}\right]$ is the selected indicator, $r_{l}$ is the time difference correlation coefficient, that

$$
r_{l}=\frac{\sum_{t=t^{\prime}}^{n_{l}}\left(x_{t+l}-\bar{x}\right)\left(y_{t}-\bar{y}\right)}{\sqrt{\sum_{t=t^{\prime}}^{n_{l}}\left(x_{t+l}-x\right)}{ }^{2} \sum_{t=t^{\prime}}^{n_{l}}\left(y_{t}-y\right)} t^{\prime}=\left\{\begin{array}{cc}
1 & l \geq 0 \\
1-l l<0
\end{array} l=0, \pm 1, \pm 2, \ldots\right.
$$

In this formula, $\bar{x}, \bar{y}$ is the variance of time series $x, y . l$ time difference or delay period. When $l$ is negative expressed in advance, said in the positive lag. $n_{l}$ is the number of data after data alignment. After calculating, we choose the maximum time difference correlation coefficient of absolute value, and take it as the lead or lag time of $x$ and $y$. Usually, when $-3 \leq l \leq 3$, this index is determined to be able to reflect the trend of the electricity market boom and the consistency index; when $l>3$, this index is determined to lag behind the trend of the electricity market boom; when $l<-3$, this indicator is the first to use electricity market sentiment changes to predict the trend of the economic changes in the leading indicators.

\subsection{Using the time difference correlation analysis method to screen the electricity market boom index}

In this paper, we take the whole society electricity consumption by power index, urban and rural consumption total electricity consumption, agriculture, forestry, animal husbandry and fishery, heavy industrial use of electricity, light industrial electricity consumption, consumption in the construction industry, transportation of electricity, commercial electricity consumption, consumption of textile industry, petroleum processing power consumption, pharmaceutical manufacturing consumption as boom analysis indicators. The whole society 
electricity can reflect the real situation of the development of a regional power market to a large extent, so it is regarded as the benchmark index. Analysis using the seasonal adjusted index data.

The month in which the maximum of the absolute value of the relationship between the selected index and the reference index is the month in which the index is the first or lagging of the index, use the positive, negative or zero in the first or the lag period to classify the discrimination index.

\section{Preparation of Electricity Market Sentiment Index}

\subsection{Preparation and significance of diffusion index}

DI (Index Diffusion) is defined as the number of indicators in the expansion of the number of indicators of the total number of selected indicators.

The calculation method for DI is:

$$
D I=\frac{\sum_{i=1}^{N}\left[D\left(A_{i}-A_{i-3}\right) W_{i} f\left(u_{i}\right)\right]}{\sum_{i=1}^{N} f\left(u_{i}\right)} \times 100 \%
$$

In the above formula,

Wi: the weight of the I index.

$$
D\left(A_{i}-A_{i-3}\right)= \begin{cases}1, & A_{i}-A_{i-3}>0 \\ 0, & A_{i}-A_{i-3} \leq 0\end{cases}
$$

\subsection{Preparation of composite index and its significance}

Composite index, according to similar indicators in the sequence of cyclic fluctuation degree, consider the sequence in overall economic activity in the importance weighted (sometimes not weighted) compiled comprehensive and, to reflect the overall economic cycle fluctuation degree index.

In this paper, we use the principal component analysis method in the multivariate statistical analysis to analyze the composite index, using the linear least square method to fit the growth rate of the main components and the electricity consumption. The fitting coefficients are inverse transformed to get the weight of each economic index.

\section{Example}

This paper chooses the whole society electricity consumption, electricity consumption of urban and rural residents, agriculture, forestry, animal 
husbandry and fishery, heavy industry, light industry, construction, transportation, commercial, textile industry, oil processing, pharmaceutical manufacturing consumption index of Guangxi from December 2014 to January 2012 , by means of qualitative and quantitative analysis, the index system of Guangxi electric power prosperity analysis system is established.

When the data is removed from the season and after the holiday adjustment is completed, to calculate the total consumption of urban and rural residents' life and the whole society electricity consumption, Get the results as shown in Table 1.

From table 1 we can see, when $\operatorname{Lag}($ Antecedent number)=1, The absolute maximum of the time difference correlation coefficient is 0.613 , so we can determine the total consumption of urban and rural residents' life is a lagging indicator of total social electricity consumption. In the same way we can get the calculation results of other indicators as shown in table 2 .

As can be seen from the table 2, the time difference of the absolute value of the total electricity consumption, the commercial electricity consumption and the textile industry power consumption is 1,1 and 2 respectively, so we can be sure that these three indicators are reflected in the electricity market changes lag indicators. When the time correlation coefficient of transportation industry and oil processing industry is the largest, the number of lead or lag period is -1 , therefore these two indicators are the leading indicators of the change of the electricity market. And agriculture, animal husbandry and fishery use of electricity, heavy industrial power consumption, light industrial power consumption, construction industry and pharmaceutical manufacturing power consumption of these 5 indicators, when their time difference correlation coefficient is maximum, the corresponding lead or lag time is 0 , so it can be considered to be recognized as the consistency index. 
Table1. Cross correlations

\begin{tabular}{lll}
\hline Lag & Cross Correlation & Std.Error(a) \\
\hline-7 & 0.082 & 0.137 \\
-6 & 0.234 & 0.136 \\
-5 & 0.456 & 0.135 \\
-4 & 0.472 & 0.134 \\
-3 & 0.457 & 0.132 \\
-2 & 0.261 & 0.131 \\
-1 & 0.285 & 0.130 \\
0 & 0.567 & 0.129 \\
1 & 0.613 & 0.130 \\
2 & 0.429 & 0.131 \\
3 & 0.311 & 0.132 \\
4 & 0.211 & 0.134 \\
5 & 0.094 & 0.135 \\
6 & 0.199 & 0.136 \\
7 & 0.328 & 0.137 \\
\hline
\end{tabular}

Select the first set of indicators defined by the calculation of screening, using the principal component analysis in SPSS to get the composition and contribution rate. From the component matrix can be seen, Let $\mathrm{L}$ as the first principal component, $\mathrm{x}_{1}, \mathrm{x}_{2}$ respectively, said the transportation industry, the oil processing industry, there are

$$
L=0.814 x_{1}+0.814 x_{2}
$$

In the same way, the composition and contribution rate of the principal component analysis of the consistent index group were obtained. Because of the first principal component and the second principal component of the cumulative contribution rate of more than $80 \%$, so we let $\mathrm{L} 1$ as the first principal component, L2 is the second principal component, $x_{1}, x_{2}, x_{3}, x_{4}, x_{5}$ respectively in farming, forestry, animal husbandry and fishery, heavy industry, light industry, construction industry, medicine manufacturing industry, there are:

$$
\begin{aligned}
& L_{1}=0.790 x_{1}+0.507 x_{2}+0.076 x_{3} \\
& +0.914 x_{4}+0.815 x_{5}
\end{aligned}
$$




$$
\begin{aligned}
& L_{2}=-0.314 x_{1}+0.805 x_{2}+0.952 x_{3} \\
& -0.066 x_{4}-0.211 x_{5}
\end{aligned}
$$

Table 2 The maximum value of the first or the lagging period and the mutual correlation coefficient of each industry

\begin{tabular}{lll}
\hline $\begin{array}{l}\text { Electricity consumption } \\
\text { index (power consumption) }\end{array}$ & $\begin{array}{l}\text { Number of antecedent or lag } \\
\text { period }\end{array}$ & $\begin{array}{l}\text { Maximum number of mutual } \\
\text { relations }\end{array}$ \\
\hline Urban and rural total & 1 & 0.613 \\
Agriculture, forestry, animal & 0 & 0.588 \\
husbandry, fishery & & \\
heavy industry & 0 & 0.89 \\
light industry & 0 & 0.596 \\
Construction business & 0 & 0.686 \\
Transportation & -1 & 0.569 \\
Business & 1 & 0.547 \\
Textile industry & 2 & -0.397 \\
Petroleum processing & -1 & 0.57 \\
Pharmaceutical & 0 & 0.422 \\
manufacturing & & \\
\hline
\end{tabular}

In the same way, the principal component analysis component of the lagging indicator group is provided with $\mathrm{L}$ as the first principal component, $\mathrm{x}_{1}$, $\mathrm{x}_{2}$ and $\mathrm{x}_{3}$ said the urban and rural residents with electricity, electricity consumption, electricity consumption in the textile industry, there are:

$$
L=0.920 x_{1}+0.945 x_{2}+0.576 x_{3}
$$

Diffusion index and composite index method were used to calculate the first diffusion index ,composite index, uniform diffusion index, uniform composite index, lagging diffusion index and lagging composite index of the electricity market in Guangxi 2012-2014,in order to reflect the regional electricity market, the overall change trend and the degree of volatility, and provide scientific basis for the relevant departments in advance to judge the situation of supply and demand of the market.

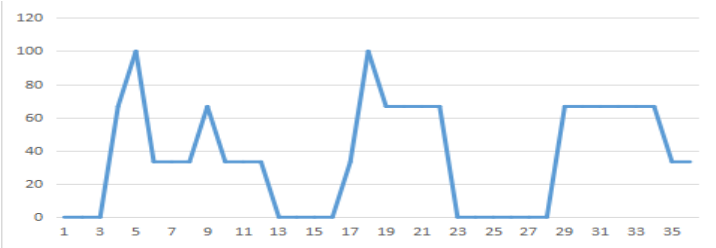

Fig. 2. Guangxi 2012-2014 years ahead of the spread of the index fluctuations 
According to the principle of the diffusion index, we first observe the diffusion index of leading indicators (as shown in Figure 2). Due to advance indicators can be at least March early warning, we can observe the first diffusion index in the annual 3-5 months, that is, the actual 6-8 months, the summer time diffusion index was more than $50 \%$, that is to say that the period of the entire electricity market is in a state of prosperity, and the high level of operation. Especially in 2013 8-10 month data are high.

Over the same period of the leading composite index (as shown in Figure 3), generally presents a fluctuation and has the trend of a rise, by its twists and turns, it can be observed that the impact of the season on the use of electricity, in spring and summer, the electricity gradually increased, and entered the peak period. On the other hand, due to high power consumption in the summer, adjustments to decrease the power consumption is made, but the overall level is still higher than the last year with the radio Valley, which shows that the development of the electricity market itself is healthy.

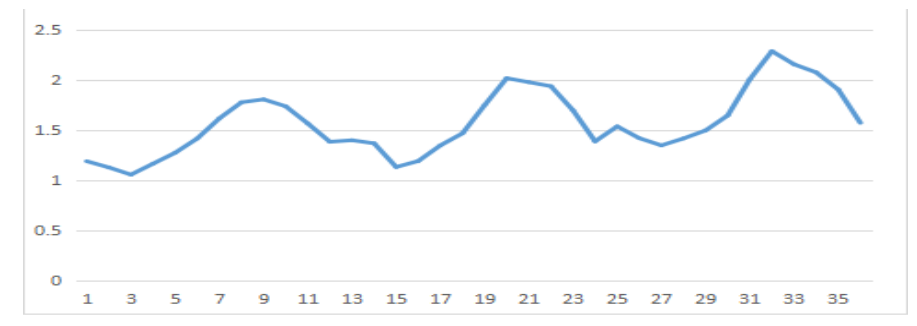

Fig. 3. Guangxi 2012-2014 leading composite index fluctuations

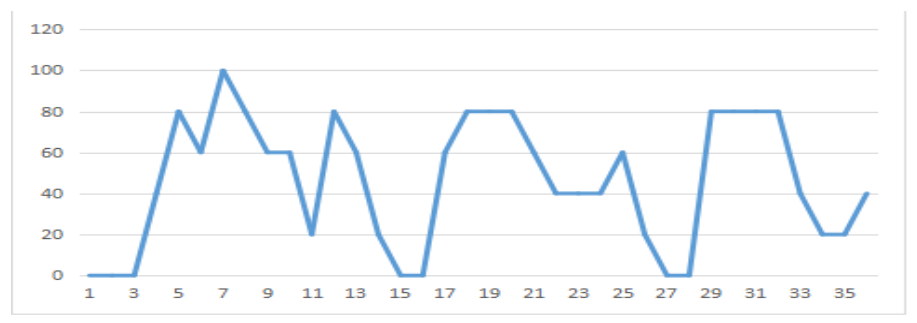

Fig. 4. Guangxi 2012-2014 uniform diffusion index fluctuations

From 2012 to 2014, the consistency index of the consistency index of figure 4 , we can see that most of the time point of the diffusion index is located in the economic zone, diffusion index is higher than $50 \%$, and has high level of operation, this reflects the electricity market during this period of time in a state of prosperity. Even if there are individual months (3-4 months) of the diffusion 
index is less than $50 \%$, it will quickly return to the previous level, did not affect the long-term prosperity of the market state. The consistency index in Figure 5 presents a slowly rising trend in the whole, this trend reflects the impact of seasonal factors in the electricity market.

Generally speaking, In 2012 and 2014, the similarity was slightly higher, and there was a significant seasonal effect. The lagging composite index (shown in Figure 6) is in a state of oscillation as a whole, the summer of the year rises to the highest level of the year, and then quickly down to a slightly higher than the level of the spring and summer of the year. And then continue to slow the rise of shock, at the end of the year to form a slightly below the level of the peak of the summer synthetic index. This trend mainly reflects the lagging indicators, Especially the urban and rural residents' electricity consumption index is affected by the seasonal factors. (mainly concentrated in the summer and the end of the Spring Festival period, this period of time consumption of urban and rural residents increased significantly), the impact of rising cost of power generation enterprises is small, and its development is stable.

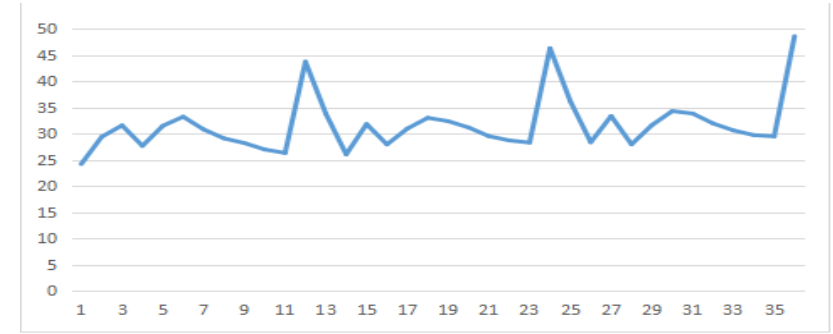

Fig. 5. Guangxi 2012-2014 uniform composite index volatility chart

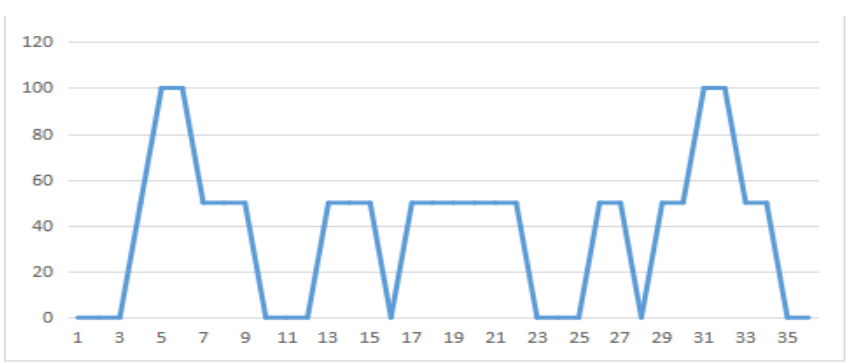

Fig. 6. Guangxi 2012-2014 lag diffusion index fluctuations 


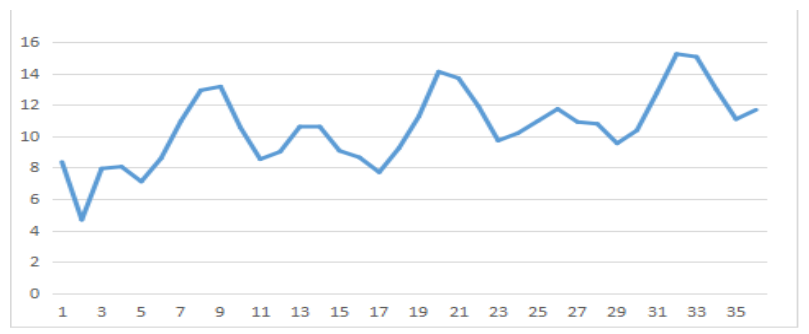

Fig. 7. Guangxi 2012-2014 lag diffusion index fluctuations

\section{Summary}

In this paper, the prosperity analysis is applied to the electricity market, carry out the research and application of related theory, electricity market sentiment has been gradually established, explore the new method of electricity market sentiment analysis and new ways, try to improve operation efficiency in the electricity market environment for power enterprises, effectively guard against the risks to provide quantitative basis, come to a conclusion as follows:

-In the extraction and use of the index data of the electricity market, because of the influence of the seasonal and irregular factors, direct analysis and calculation of time series are often not stable and cannot get reliable results. We need to preprocess the original data, through the X-12-ARIMA seasonal adjustment, to build a stable indicator of changes in the data. After trial calculation, it is found that these sequences can truly reflect the long-term trend of the electricity consumption and the fluctuation range of the power consumption.

-The time difference correlation analysis method is applied to the multi index and the large scale data screening and classification, By calculating and analyzing the time difference correlation coefficient between different sectors of electricity consumption and total electricity consumption, the different indicators are included in the first index group, the consistency index group and the lagging indicator group. And in this calculation method, the model is simple, the process is convenient, the result is accurate, so that can provide a good data support for the next step in the preparation of the electricity market sentiment index.

-By the method of diffusion index and principal component analysis, the electricity market sentiment index was compiled, and the variation period of the electricity market was quantified, which confirmed that there was a certain periodicity in the regional electricity market. 


\section{References}

1. Chen Lei, Wu Guizhen, Gao Tiemei. Principal component analysis with the fluctuations [J]. Quantity economy and technology economy research, 1993, 7:32 55.(In Chinese)

2. Yang Jinmei. Research on analysis method of electricity market sentiment analysis [D]. Beijing: Department of electrical engineering, Tsinghua University, 2004. (In Chinese)

3. Gao Tiemei, Liang Yunfang. A comprehensive analysis of the survey data of China's industrial boom [J]. prediction, 2002,4: 5 9. (In Chinese)

4. Lai Fuping. Research and empirical analysis of industrial enterprise boom index [D]. Guangzhou: Jinan University, 2005. (In Chinese)

5. Li Junrui. Analysis of the oil and gas industry boom in China [D]. Jilin: Jilin University, 2006. (In Chinese)

6. Huang Dejun. Using principal component analysis method to compile comprehensive climate index [J]. statistics education, 2005, 2:1 2. (In Chinese)

7. Zeng Ming. Power demand side management incentive mechanism and its application [M]. Beijing: China Electric Power Press, 2002, 13 25. (In Chinese)

8. Kang Chongqing, Li Shunfu, Xia Qing. Structural analysis of electricity market and its implications for marketing [J]. automation of electric power systems, 2003, 27 (14): 27 31. (In Chinese) 